\title{
Cocaine inflicts 'Silent' damage to the heart: a case report
}

\author{
Massimo Bolognesi* and Diletta Bolognesi \\ Department of Territorial Medicine - Primary Care, Internal General Medicine - AUSL della Romagna District of Cesena, Via Ungaretti 49447521 Cesena, Italy
}

\begin{abstract}
Cocaine use is associated with both ischaemic and non-ischaemic cardiac complications. The prevalence of myocardial damage in asymptomatic addicts has been evaluated by cardiovascular magnetic resonance (CMR), which allows non-invasive detection of myocardial oedema and fibrosis. Here the authors describe an emblematic case report.
\end{abstract}

\section{Introduction}

Cocaine use has been steadily increasing and there are many individuals who have recently made use of cocaine, and most of these are met for cocaine dependence or abuse [1].

The DSM-IV-TR psychiatric diagnoses for cocaine abuse and cocaine dependence were replaced by one diagnosis, cocaine use disorder, in DSM-5 [2]. Although the crosswalk between DSM-IV and DSM-5 disorders is imprecise, cocaine dependence is approximately comparable to cocaine use disorder, moderate to severe subtype, while cocaine abuse is similar to the mild subtype [3].

Cardiopulmonary symptoms are the most frequent complaints in cocaine users who seek medical help, with chest pain being the most frequent symptom [4]. Cocaine acutely increases heart rate, blood pressure, and systemic vascular resistance by increasing adrenergic activity in the heart, and indirectly via the Central Nervous System (CNS) [5]. The increased myocardial oxygen demand, coupled with decreased coronary blood flow from vasospasm and vasoconstriction, can cause acute myocardial infarction, even in young individuals without atherosclerosis. Cocaine use is a factor in about one-quarter of non-fatal heart attacks in persons younger than 45 [6]. Cocaine use increases risk for cardiac arrhythmias and sudden death [7]. Chronic use is associated with left ventricular hypertrophy, cardiomyopathy, myocardial fibrosis, and myocarditis [8]. Cocaine use can lead to addiction and adverse physical effects, such as stroke and cardiac arrest. Despite being asymptomatic, heavy cocaine users have a high prevalence of cardiac damage as seen by cardiac magnetic resonance imaging. Cardiovascular magnetic resonance (CMR) is the gold standard to discover silent myocardial damage that usually was not apparent with other diagnostic tools, such as troponin or natriuretic peptide-wise or electrocardiography, echocardiography, or exercise stress testing, as reported in Heart with a study conducted on a group of asymptomatic chronic cocaine users [9]. Here the authors describe an emblematic case report.

\section{Our experience}

This report concerns a 54-year-old gentleman who was admitted to the psychiatric department about 5 years ago due a delusional disorder caused by substance abuse (cocaine). This patient has a positive family history for ischemic heart disease, psychosis, and type 2 diabetes mellitus. For over five years he has been treated for diabetes and has been on pharmacological therapy to control his high blood pressure. Furthermore, he suffer from a bicuspid aortic valve with ascending aortic dilatation. For these reasons he routinely undergoes various cardiovascular investigations, ie two-dimensional trans-thoracic echocardiography (2D TT), cardiac computed tomography angiogram (CCTA) and, more recently cardiac magnetic resonance (CMR). The last echocardiography examination not only confirmed the presence both of bicuspid aortic valve with mild steno-insufficiency and a moderate dilatation of the ascending aorta up to $45 \mathrm{~mm}$, but also showed a marked left ventricular hypertrophy with areas of hyperechogenicity suggestive for fibrosis at the basal segment of the septal and inferiorlateral wall (Video 1). Resting electrocardiogram (ECG) abnormalities were not observed, and 2D TT echocardiography showed no regional wall-motion abnormalities with normal global LV function. There

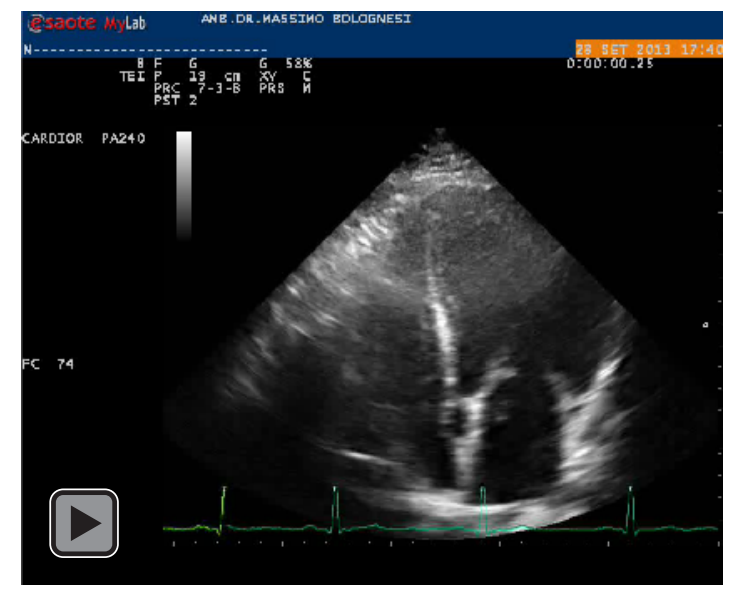

Correspondence to: Massimo Bolognesi, Department of Territorial Medicine - Primary Care, Internal General Medicine - AUSL della Romagna District of Cesena, Via Ungaretti 49447521 Cesena (Italy) +390547645074; E-mail: massbolo1@tin.it

Key words: cocaine addicts, silent myocardial damage, echocardiography, cardiac magnetic resonance

Received: April 24, 2015; Accepted: May 24, 2015; Published: May 27, 2015 
were no arrhythmias, angina, or other evidence of inducible ischemia at stress testing or 24-hour Holter monitoring. All other biomarker tests were negative. A previous coronary computed tomography angiography showed diffuse coronary atherosclerosis without critical narrowing of the epicardial vessels. Consequently a CMR was performed. This cardiovascular imaging confirmed the presence of intramyocardial areas of fibrosis (with gadolinium enhancement), i.e. appearance of late gadolinium enhancement in intra-myocardial distribution at the basal interventricular septum, lateral wall and basal medium and basal inferior wall (Video 2-3), suggestive of alterations in the microcirculation and of myocardial damage due to chronic use of cocaine.

\section{Discussion and conclusions}

Cocaine is among the most commonly used illicit recreational drugs worldwide. Since casual use of cocaine may be associated with acute or chronic cardiovascular toxicity, the large numbers of exposed individuals represent a reservoir of patients who may present with sequelae related to the cardiovascular system [8]. CMR is the best cardiovascular imaging technique for the assessment of ventricular dimensions and function, with high accuracy and reproducibility and reference values established for both the left [10] and right ventricles [11]. CMR can provide a comprehensive evaluation of the cardiovascular system and has a formidable capability for tissue characterization, which has been applied to the assessment of several myocardial disease such as cardiomyopathies $[12,13]$. Thus, the cardiovascular history should include questions about cocaine use, specifically focusing on the occurrence of symptoms associated with coronary heart disease. A high prevalence of cardiac damage in asymptomatic cocaine addicts can be suspected by echocardiography and found by CMR examination. The
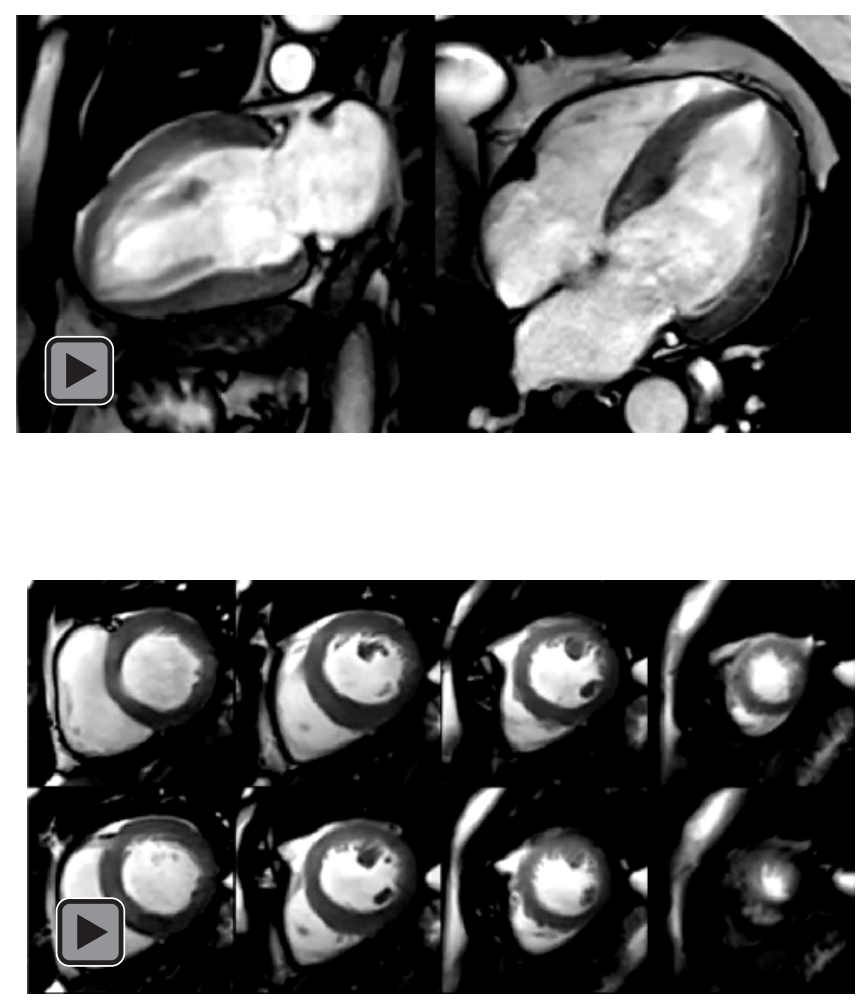

presence of coronary artery disease is not always indispensable for the development of myocardial damage and late gadolinium enhancement (LGE). However, myocardial infarction can occur as a result of coronary thrombus in patients with otherwise normal coronary arteries [14]. As a recent report of literature this case has showed in a casual cocaine user multiple spots of midmyocardial LGE taken as potential evidence of cocaine-induced vasoconstriction of coronary arterioles [15]. Some of the mid-myocardial areas of LGE found might well account for coronary vasospasm or small areas of thrombus occlusion, bearing in mind that this pattern is also observed in a number of cardiomyopathies [16]. In conclusion, as Acquaro et al. [9] report there are limited data on the effects of cocaine in asymptomatic users, therefore more evidence is needed.

\section{References}

1. Volkow ND (2009) National Institute on Drug Abuse. Cocaine: Abuse and Addiction. Washington, DC: US Department of Health and Human Services.

2. DSM-5 Diagnostic and Statistical Manual of Mental Disorders, (5th Edn.), Edited by American Psychiatric Association

3. Compton WM, Dawson DA, Goldstein RB, Grant BF (2013) Crosswalk between DSMIV dependence and DSM-5 substance use disorders for opioids, cannabis, cocaine and alcohol. Drug Alcohol Depend 132: 387-390. [Crossref]

4. McCord J, Jneid H, Hollander JE, de Lemos JA, Cercek B, et al. (2008) American Heart Association Acute Cardiac Care Committee of the Council on Clinical Cardiology. Management of cocaine-associated chest pain and myocardial infarction: a scientific statement from the American Heart Association Acute Cardiac Care Committee of the Council on Clinical Cardiology. Circulation 117: 1897-1907. [Crossref]

5. Afonso L, Mohammad T, Thatai D (2007) Crack whips the heart: a review of the cardiovascular toxicity of cocaine. Am J Cardiol 100: 1040-1043. [Crossref]

6. Qureshi AI, Suri MF, Guterman LR, Hopkins LN (2001) Cocaine use and the likelihood of nonfatal myocardial infarction and stroke: data from the Third National Health and Nutrition Examination Survey. Circulation 103: 502-506. [Crossref]

7. Lange RA, Hillis LD (2001) Cardiovascular complications of cocaine use. $N$ Engl J Med 345: 351-358. [Crossref]

8. Schwartz BG, Rezkalla S, Kloner RA (2010) Cardiovascular effects of cocaine. Circulation 122: 2558-2569. [Crossref]

9. Aquaro GD, Gabutti A, Meini M, Prontera C, Pasanisi E, et al. (2011) Silent myocardial damage in cocaine addicts. Heart 97: 2056-2062. [Crossref]

10. Maceira AM, Prasad SK, Khan M, Pennell DJ (2006) Normalized left ventricular systolic and diastolic function by steady state free precession cardiovascular magnetic resonance. J Cardiovasc Magn Reson 8: 417-426. [Crossref]

11. Maceira AM, Prasad SK, Khan M, Pennell DJ (2006) Reference right ventricular systolic and diastolic function normalized to age, gender and body surface area from steady-state free precession cardiovascular magnetic resonance. Eur Heart J. 27: 28792888. [Crossref]

12. Mahrholdt H, Wagner A, Judd RM, Sechtem U, Kim RJ (2005) Delayed enhancement cardiovascular magnetic resonance assessment of non-ischaemic cardiomyopathies. Eur Heart $J$ 26: 1461-1474

13. Assomull RG, Prasad SK, Lyne J, Smith G, Burman ED, et al. (2006) Cardiovascular magnetic resonance, fibrosis, and prognosis in dilated cardiomyopathy. $J$ Am Coll Cardiol 48: 1977-1985. [Crossref]

14. Robaei D, Grieve SM, Nelson GC, Bhindi R, Figtree GA (2010) Cocaine-induced epicardial coronary artery thrombosis resulting in extensive myocardial injury assessed by cardiac magnetic resonance imaging. Eur Heart $J$ 10:14. [Crossref]

15. Buchholz S, Grieve SM, Maher R, Figtree GA (2010) Cocaine-induced myocardial injury seen as multiple mid-wall foci of late enhancement by contrastenhanced cardiac magnetic resonance imaging. Eur Heart J. 31:1422.

16. Kazimir M. Cocaine-related cardiomyopathy. Medscape.

Copyright: (C2015 Bolognesi M. This is an open-access article distributed under the terms of the Creative Commons Attribution License, which permits unrestricted use, distribution, and reproduction in any medium, provided the original author and source are credited. 